\title{
Compte-rendu. La Séparation de Claude Simon : un texte précurseur
}

Dominique Viart

\section{OpenEdition}

1 Journals

Édition électronique

URL : https://journals.openedition.org/ccs/3166

DOI : $10.4000 /$ ccs. 3166

ISSN : 2558-782X

Éditeur :

Presses universitaires de Rennes, Association des lecteurs de Claude Simon

\section{Édition imprimée}

Date de publication : 3 septembre 2020

Pagination : 273-283

ISBN : 978-2-7535-8065-7

ISSN : $1774-9425$

Référence électronique

Dominique Viart, "Compte-rendu. La Séparation de Claude Simon : un texte précurseur », Cahiers

Claude Simon [En ligne], 15 | 2020, mis en ligne le 03 septembre 2021, consulté le 05 décembre 2022

URL : http://journals.openedition.org/ccs/3166; DOI : https://doi.org/10.4000/ccs.3166 


\title{
LA SÉPARATION DE CLAUDE SIMON : UN TEXTE PRÉCURSEUR
}

\author{
Dominique VIART
}

Claude Simon, La Séparation (1963), postface de Mireille Calle-Gruber, Les éditions du Chemin de Fer, 2019.

Paraît aux éditions du Chemin de fer, une pièce de Claude Simon, La Séparation. Il s'agit d'un texte joué en 1963 au Théâtre de Lutèce dans une mise en scène de Nicole Kessel. Le prétexte dramaturgique reprend la situation du roman L'Herbe, paru cinq ans auparavant aux éditions de Minuit : deux couples discutent et se disputent: Georges et sa femme Louise d'une part; Sabine et Pierre, les parents de Georges, de l'autre, tandis que, hors scène, agonise Marie, la vieille sœur de Pierre, dont une garde-malade, " petite vieille contrefaite et bossue ", apparaît pour donner des nouvelles. Le dispositif scénique est très précis. Bien loin de l'abstraction moderniste d'En attendant Godot, dont le décor se résume au seul arbre conçu par Giacometti, celui de La Séparation est substantiel, et rigoureusement fixé : « deux cabinets de toilette » séparés par une cloison, avec des «murs en carreaux de faïence décorés à la japonaise de branches de cerisiers en fleurs et d'oiseaux ", explique la didascalie initiale; un mobilier tout aussi précisément énuméré : lavabo, glace, fauteuil et chaise en rotin d'une part; vieux meubles sans style recouverts de tissus décolorés, pouf, coiffeuse, chaise basse ripolinée, liseuse à jupon de l'autre, paravents de part et d'autre, etc.

Un tel " réalisme " mimétique intrigue. Il propose un décor de théâtre de boulevard que l'on n'attend guère sous la plume de l'auteur. Ou bien est-ce le difficile exercice qui le conduit à passer d'une pratique romanesque très attentive aux objets, aux meubles et aux aménagements toujours si longuement 
décrits, au dépouillement d'une scène théâtrale que l'on attendrait sans doute plus économe de la part de Simon? Comment faire entrer la richesse d'un roman aussi profus que L'Herbe sur la scène plus restreinte du théâtre? La réponse est peut-être ailleurs. Je tenterai de l'esquisser plus loin. Mais il faut d'abord savoir ce qu'il en est du théâtre dans l'œuvre de Simon et de cet hapax que constitue La Séparation. Car si l'on connaît bien désormais, grâce surtout aux travaux de Bérénice Bonhomme, l'intérêt que Claude Simon portait au cinéma ${ }^{1}$, ainsi que ses tentatives dans ce domaine, dont témoignent le film L'Impasse (Die Sackgasse) réalisé en 1975 par Georg Bense et Peter Brugger sur un scénario de l'écrivain d'après Triptyque, et le découpage de La Route des Flandres qui clôt Le Jardin des Plantes, on en sait moins sur son goût pour le théâtre.

De nombreux passages de ses romans y font cependant allusion, qu'il s'agisse des spectacles auxquels il est donné au narrateur d'assister ou, plus encore, des références métaphoriques que le texte convoque, par exemple les portraits de tel personnage maternel en tragédienne trop fardée. Car le monde du théâtre sert souvent de comparatif dans les discours du narrateur qui voit les individus jouer un rôle, affecter une posture, construire leur personnage social. Pour Claude Simon, le monde apparaît comme une comédie, un univers factice, où chacun se donne en représentation. L'exagération du tempérament espagnol, volontiers baroque et spectaculaire, se prête aisément à ces formulations. Et l'écrivain a l'œil pour dénoncer la comédie sociale dans laquelle chacun se fige dans une figure qu'il tente de composer, pour luimême et pour autrui, avec une " héréditaire propension au théâtral " (Tram., p. 1293). On se souvient également des mentions plus directes qu'il fait de cet art lorsqu'il ironise sur les pièces écrites pendant l'Occupation : " la mode au théâtre est à ces travaux d'universitaires époussetant laborieusement les vieux mythes et où, vêtues de chlamydes, de péplums, chaussées de cothurnes, les incarnations fardées du Pouvoir, du Droit et de la Justice s'affrontent à coup d'allusions et de formules à double sens applaudies au passage par un public complice» (JP, p. 1149).

Il faut aussi compter avec le récit sarcastique que Simon donne dans Le Jardin des Plantes de la représentation de la pièce de Picasso, Le Désir attrapé par la queue, à laquelle il assista le 19 mars 1944 à l'invitation de Dora Maar, dans

1. Voir Bérénice Bonhomme, Triptyque de Claude Simon : du livre au film : une esthétique du passage, Presses de l'université de Paris-Sorbonne, 2005; Claude Simon, la passion cinéma, Presses universitaires du Septentrion, 2010; Claude Simon : une écriture en cinéma, Oxford - New York, Peter Lang, 2010. 
l'appartement de Michel et Zette Leiris. Interprétée par Leiris et sa femme, par Sartre, Beauvoir, Dora Maar, Raymond Queneau, Jacques-Laurent Bost et quelques autres, la pièce avait été mise en scène à cette occasion par Albert Camus et fut jouée en présence de Bataille, Barrault, Braque, Brassaï, Lacan, Reverdy, Cécile Eluard, Valentine Hugo... tout un aréopage intellectuel et artistique entremêlé. Simon, plus fruste, moins bien habillé et fraîchement arrivé de sa lointaine province, se décrit comme décalé dans cette "volière bienséante et feutrée " puis " jacassant, assourdissante " (JP, p. 1151, 1152). À chaque fois donc, le théâtre apparaît comme l'instrument ou l'occasion d'un développement critique, voué à dénoncer les artifices, les exagérations, les poses et les postures, la vanité mondaine.

La publication de La Séparation montre toutefois que ce n'est pas là le seul mode de présence du théâtre dans l'œuvre de l'écrivain, mais que Simon s'y est intéressé au point de vouloir en écrire. Il semble même que ce fut une tentation précoce, nouée autour d'un goût pour le dialogue comique dont plusieurs manuscrits parmi les plus anciens conservés portent trace - et que l'on retrouve parfois dans les romans. Il est dommage que la postface livrée avec le texte et censée éclairer la dimension dramaturgique de l'œuvre n'en fasse pas état. Du reste, cette postface, imprimée en des caractères si petits qu'elle en est rendue quasiment illisible (à se demander si l'éditeur, désolé de son piètre intérêt, n'a pas voulu économiser sur le nombre de feuillets), n’informe pas beaucoup sur la relation que Claude Simon a pu nouer avec l'univers dramaturgique. Or se trouve notamment dans les papiers de l'écrivain une brève saynète italienne, intitulée "L'Altesse royale " qui met en scène, de manière assez burlesque, l'affrontement du théâtre et du roman.

Entièrement dialoguée, elle présente deux personnages : un dramaturge italien un peu ridicule, auquel on doit " quarante-sept piècés dé théâtré rien qué sur des sujets éternels ", mais dont, hélas (?), la "veiné sé tarit ", et un écrivain plus posé, voire circonspect, auteur de deux romans et d'un essai. À supposer que cet écrivain soit inspiré de la figure de Simon lui-même, cela situe le manuscrit du début des années 50, après la parution du Tricheur, de Gulliver et de La Corde raide: de 1953 sans doute, ce que l'état du papier semble confirmer. C'est, pour Simon, l'occasion de mesurer les deux genres - théâtre et roman - leurs avantages et leurs inconvénients respectifs. Le roman, soutient l'écrivain, est plus libre : "On peut développer ce qui vous plaît ". Alors que "le théâtre, c'est terrible! C'est ouniquement oun affairé d'argent! ». Est-ce pour cela, la liberté de l'un, le coût de l'autre, que 
Simon privilégia le roman? On peut le penser. Mais penser aussi que cette liberté narrative lui convint mieux, d'autant qu'elle lui permettait, à côté des dialogues dont il a constamment retravaillé la forme, les insérant dans le flux même de la prose narrative, de développer ces vastes descriptions et remémorations qui sont sa manière même, et auxquelles évidemment le théâtre pouvait difficilement faire place.

Il n'en demeure pas moins qu'il fut tenté par l'écriture dramaturgique, et qu'une pièce de sa main, La Séparation donc, fut représentée en 1963 à Paris. D'où lui est venue l'idée de l'écrire? S'agit-il d'une décision de sa part ou d'une commande? Est-ce Simon lui-même qui a proposé son texte à Nicole Kessel qui l'a mis en scène, ou Nicole Kessel qui lui a demandé un texte? Lui aurait-elle fait part de son désir de transposer L'Herbe sur la scène théâtrale? Cruellement déficiente à cet égard, la postface n'en dit mot. En fait, l'actrice, nièce de l'écrivain Joseph Kessel, n'aura de toute sa carrière mis en scène que deux pièces : deux ans avant La Séparation, elle adapte Les Nuits blanches de Dostoïevski, dont elle avait joué en 1959 Les Possédés dans une mise en scène d'Albert Camus. Des Nuits blanches à La Séparation, le lien n'est pas impossible : un couple qui ne parvient pas à se constituer d'une part; un autre qui se délite d'autre part et derrière ces histoires de sentiment et de difficile ajointement des êtres, le sens de l'attente, l'absence, la mort, la mélancolie. Cette mélancolie dont Simon rappellera justement dans Le Jardin des Plantes le sens dostoïevskien que ce mot prend pour lui. Nicole Kessel s'avère en ces années sensible au théâtre qui s'invente alors sous la plume des écrivains de l'avantgarde. Elle joue des pièces de Ramon del Valle-Inclàn ou de Jean-Pierre Faye sous la direction de Roger Blin, metteur en scène de Beckett et de Genet; Genet dont Blin avait présenté Les Nègres, dans ce même petit Théâtre de Lutèce (une salle de 350 places à peine, sans coulisses) en 1959.

Et sans doute est-ce bien ainsi que Nicole Kessel s'est approprié le texte de La Séparation, comme la rencontre d'un univers dostoïevskien et d'une préoccupation plus moderne du langage, lieu effectif du drame existentiel, lorsqu'aucune action ne vient scander la pièce de ses péripéties. À rebours, donc, du théâtre bourgeois que la didascalie initiale fait attendre, et auquel ont cru assister des critiques comme Michel Déon ou Gabriel Marcel, si l'on en croit les comptes rendus qu'ils firent de la représentation. Claude Simon assiste aux répétitions et à la première, qui le déçoit, et part en voyage aux Pays-Bas pour une tournée de conférences. Sa compagne Réa lui écrit que la pièce est un "succès " mais, précise-t-elle, "dans le malentendu ». Formule 
importante et révélatrice. Quelque chose en effet s'est grippé. L'intention n’a pas tenu sa promesse. On peut émettre une hypothèse : celle d'une regrettable erreur de distribution. Deux acteurs n'avaient peut-être pas leur place dans une telle pièce, deux acteurs dont le jeu pouvait en déformer le sens. Bavarde et grandiloquente, la postface, qui ressasse des informations connues de tous sur ce que fut le "nouveau roman ", la photo de Dondero devant le 7 rue Bernard-Palissy et autres lieux communs de l'histoire littéraire, ne nous informe guère sur ces circonstances de la mise en scène et de la distribution. Or c'est là sans doute que la déconvenue de Simon prend sa source.

Si Marc Eyraud, qui joue Georges, n'est pas encore le grand acteur qu'il est appelé à devenir, interprète de Buńuel, de Rivette, de Varda, de Costa-Gavras, de Rohmer... son talent et son exigence sont déjà incontestables. Il travaille pour Jouvet, Pitoëff, Camus, Régy. Nul doute qu'il ait été un Georges pleinement convaincant, tout comme Nicole Kessel devait l'être elle-même dans le rôle de Louise. Mais les autres? Marcel Journet interprète Pierre. C'est un acteur de comédies musicales. Il a joué à Broadway. Il joue dans des opérettes (Pacifico), il joue Guitry (La Fin du monde). Son univers n'est manifestement pas celui de Simon, qui souhaite plutôt des voix monocordes, retenues sinon abattues. Et l'on aurait surtout aimé en savoir plus sur le sentiment de Claude Simon à l'égard de l'actrice principale, Alice Cocéa, à laquelle fut confié le rôle de Sabine. Sa présence en haut de l'affiche étonne en effet à double titre, artistique et politique : venue, elle aussi, de l'opérette, Alice Cocéa a récupéré durant l'Occupation le Théâtre des Ambassadeurs qu'Henri Bernstein avait dû abandonner pour fuir le sort réservé aux Juifs. Elle y fut accueillante aux Allemands, moins sans doute par obligation que par sympathie, puisqu'elle a monté et interprété, en Suisse d'abord, puis à Paris en 1957, Bérénice ou la reine de Césarée de Brasillach.

On sait que cela donna lieu à de violentes protestations du Comité d'Action de la Résistance et des associations juives, et suscita des polémiques politiques et judiciaires qui alimenteront la chronique des années 57 et 58, d'autant plus que Cocéa, alors même que la direction du théâtre souhaitait abandonner les représentations, assigna celle-ci en justice pour les prolonger coûte que coûte, assurant contre toute évidence que Brasillach faisait l'éloge des Juifs... La Séparation est l'une des dernières pièces qui la voit monter en scène. Comment Simon a-t-il pu accepter qu'une telle actrice tienne la tête d'affiche de sa pièce? La postface ne nous en dit rien. À peine y lit-on que les outrances boulevardières de Cocéa firent, selon la presse, « dévier le spec- 
tacle vers le numéro d'actrice " (un peu comme cette postface elle-même, en quelque sorte, particulièrement complaisante à sa propre emphase).

Aussi la réception critique ne fut-elle guère élogieuse. Dans Le Monde du 13 mars 1963, on pouvait lire, sous la plume de Bertrand Poirot-Delpech : "Avec une constance qui tourne au conformisme, presque toutes les pièces d'essai prennent pour thème depuis quinze ans cette même décomposition des pensées, des sentiments et des mots à l'épreuve du temps, et pour exemples presque exclusifs de cette décrépitude, les mêmes scènes de vieux ménages égrotants et ergotants. Bien que cet intimisme désolé ait fait ses preuves - Tchekhov, James, Tennessee Williams, Duras, Antonioni..., - et que la dérision conjugale ou gérontologique garde tout son pouvoir de suggestion - Ionesco, Beckett, Dubillard, - Claude Simon court le risque, à les cultiver si fidèlement, d'avoir l'air de répéter un exercice d'école et qu'on lui en impute l'usure ou les limites. À qui la faute? Et alors? Et après?... ».

Le journaliste regrette que l'écrivain n'ait pas su transposer au monde théâtral ce qui faisait déjà l'intérêt de ses romans : "Il court d'autant plus ce risque que son obsession personnelle de la mémoire inorganisée ne trouve pas, pour ces débuts, une expression spécifiquement théâtrale. Le refus de toute action dramatique n'est compensé par aucune progression secrète. En fait de pièce, l'auteur tire successivement d'un de ses propres romans des lambeaux de réminiscences reliés par des recettes d'avant-garde et un numéro de comique sénile proche du Boulevard. Le romancier et le dialoguiste s'affirment, mais presque malgré l'auteur de théâtre, et à ses dépens. "Or ce " comique sénile " et ces accents de "boulevard ", le lecteur d'aujourd'hui ne les trouve guère dans le livre qu'il a sous les yeux. C'est donc bien le jeu des acteurs qui est venu les surajouter à un texte qui ne les supposait pas. Et c'est lui qui a transformé en "vaudeville " (selon un article de Force ouvrière daté du 3 avril 1963) ce qui aurait dû demeurer dans le ton de Tchekhov ou d'Ibsen.

Mais la pièce elle-même, indépendamment de cette distribution qui ne lui rendit pas justice, que faut-il en penser? En ce début des années 60, la question formelle est devenue centrale. Simon vient de publier Le Palace qui surenchérit sur la complexité de La Route des Flandres. Aussi est-ce d'abord ce qu'il met en avant dans la présentation qu'il fait de sa pièce. Plus que strictement dramaturgique, l'intention qui gouverne sa tentative théâtrale procède d'une réflexion sur la simultanéité. Non pas seulement celle des strates de passé dans la mémoire, que l'agencement de la phrase parvient à réunir, 
comme le modèle proustien (et plus lointainement celui de Chateaubriand) a déjà pu le montrer, mais celui des actions simultanées. On sait que cette question du simultanéisme fut l'une des grandes préoccupations des avantgardes au début du $\mathrm{Xx}^{\mathrm{e}}$ siècle. Les Futuristes, notamment, y ont consacré une part de leur énergie ${ }^{2}$. Le jeune peintre que fut Simon a été marqué par ces préoccupations. Sa syntaxe en témoigne. Et son désir d'en explorer les possibilités aussi. Le théâtre, qui déploie dans l'espace ce que le roman ne peut que produire dans la succession linéaire des pages, y est propice.

Le titre de la pièce, La Séparation désigne l'artifice scénique qui permet de mettre en scène cette simultanéité, de part et d'autre d'une cloison entre deux cabinets de toilette, implantée perpendiculairement à la salle : " une de mes préoccupations quand j'écris est de dire cette simultanéité des choses : le présent, le passé, l'avenir et ce qui se passe en même temps mais simplement et séparé par de l'espace... ou une cloison ", explique Simon dans des notes prises pour préparer un entretien consacré à sa pièce. "Il m'a semblé qu'une scène de théâtre était même le seul endroit (contrairement au roman où l'on est bien forcé d'énumérer les choses successivement) où pouvait se matérialiser tout naturellement cette simultanéité ». Et parce que les miroirs des cabinets de toilette sont placés à même hauteur de part et d'autre de la cloison, ils postulent virtuellement que l'image qu'ils renvoient de la femme qui s'y mire est celle de l'autre, installée de l'autre côté. La plus jeune y voit son avenir; la plus âgée sa jeunesse enfuie, selon un vertige et une angoisse temporels bien simoniens. La fin de la pièce qui donne à voir en parallèle Louise et Sabine, chacune de son côté, chacune enfermée dans son mal-être, Louise préparant son départ, Sabine se noyant dans l'alcool, la jalousie accumulée et d'hystériques récriminations, en est la meilleure manifestation.

On ne saurait toutefois s'en tenir à cette seule explication, formelle, du titre de la pièce. Car celui-ci est d'évidence à double sens (c'est une " pièce basée sur le fait que le langage est double ", note Simon dans ses manuscrits) : la "séparation " dit aussi la distance prise au sein de couples qui se défont, tout comme, six ans plus tôt, La Jalousie de Robbe-Grillet désignait à la fois un dispositif visuel et un sentiment négatif. Les deux titres sont superposables : ils sont à leur discrète manière une sorte de manifeste esthétique des recherches littéraires de l'époque : comment exprimer des affects sans sacrifier

2. Voir Dominique Viart, Jules Romains et les écritures de la simultanéité, Lille, PU du Septentrion, 1996; notamment «Esthétiques de la simultanéité " (p. 19-43) et « La simultanéité anachronique : Jules Romains vs Claude Simon » (p. 261-276). 
au psychologisme désuet du romanesque traditionnel? Par le jeu d'homonymie qu'ils convoquent, les termes de " jalousie » et de "séparation » déplacent l'affect sur des objets concrets, qui en deviennent non le symbole - ces écrivains ont en horreur tout ce qui ferait allégeance au symbolisme - mais le signe tangible.

C'est là la dimension behavioriste de leurs ouvres : car la " jalousie " est cet interstice d'une fenêtre par où le jaloux observe à la dérobée celle qu'il soupçonne, quand la "séparation " est cet espace qui se creuse entre les protagonistes en délicatesse. "Littérature objective ", écrivait Roland Barthes. Matérielle même, objectale, pourrait-on dire, en effet, dans la mesure où un objet se fait le signe d'une souffrance dont il cristallise les manifestations. $\mathrm{Or}$ - et c'est là le talent de Simon - la "séparation " dans La Séparation n'est pas ce seul artifice scénique : c'est aussi une épreuve dans la langue, un creusement dans l'inadéquation des dialogues, qui ne se répondent pas vraiment, n'entrent pas en discussion, rompent l'échange, s'égarent. Un creusement aussi, à l'intérieur du sujet, qui se trouve comme séparé de lui-même. Et le langage qui dit parfois ce que celui qui le profère pensait mais ne pensait pas dire, comme ce " on ne s'entend plus " que Georges formule au début de la pièce, croyant parler du piaillement puissant des moineaux dont le bruit couvre tout, et s'apercevant tout à coup que la formule vaut aussi pour le couple qu'il forme avec Louise.

La postface, acharnée à réhabiliter la pièce en la défigurant sous les espèces d'une tragédie expressionniste et morbide, fantasme Claude Simon en Léon Bloy ( «Une ouvre à la beauté crépusculaire, conjuguant l'effroi fasciné par la chair vouée à la charogne et une lucidité de Jugement Dernier $\left.{ }^{3} »\right)$. Sa dimension tragique aurait échappé à la critique. C'est ne pas comprendre grandchose ni à l'œuvre de Simon, ni à l'histoire du théâtre dans cette seconde moitié du $\mathrm{xx}^{\mathrm{e}}$ siècle. Rien en effet d'un tel exhibitionnisme dans le dispositif et le jeu voulus par Simon. Au contraire : avec La Séparation, l'auteur s'avère en avance sur son temps et c'est pourquoi sa pièce ne fut pas reçue comme elle aurait pu l'être. L'époque est à l'absurde et à la farce tragique : Adamov,

3. Toute attachée à métamorphoser par contresens la pièce de Simon en "tragédie grecque " (ne nommet-elle pas la scène skèné, pour faire chic?), la postface lui ajoute même une Parque, des Érynnies et des " ténèbres chtoniennes", afin sans doute de faire bonne figure et de l'associer aux reprises de la tragédie antique par Sartre, Giraudoux ou Anouilh, pourtant moquées par Simon dans Le Jardin des Plantes, sans voir précisément à quel point La Séparation se distingue fortement de telles allégeances et replie sur la banalité du monde humain le drame de l'existence. C'est que la tragédie, ça fait plus noble, et cela permet sans doute au critique de briller aux dépens de ce qu'il ne comprend pas. 
Beckett, Ionesco, Genet joués depuis dix ans sur de petites scènes gagnent enfin le haut du pavé. Barrault monte Rhinocéros en 1960, Blin reprend En attendant Godot en $1961^{4}$. La Séparation ne s'inscrit pas dans cette veine. Or, s'il est bien évidemment stupide de faire de cette pièce une tragédie antique, les ingrédients semblent en revanche rassemblés pour constituer une sorte de "drame bourgeois ", comme la critique a cru l'entendre : crise au sein du couple, jalousies, sentiments contrariés, décor vériste et contexte contemporain... Mais Simon arase le pathos qui caractérise cette forme théâtrale volontiers excessive dans ses affects. L'hystérie est le travers d'un personnage - Sabine - et non le fait de la pièce elle-même, bien au contraire. L'écrivain ne cherche ni à favoriser la dimension romanesque de l'intrigue, ni à produire quelque leçon moralisatrice.

On est donc aussi loin du mélodrame et du vaudeville que du symbolisme exhibitionniste et décadent. Avec plus de dix ans d'avance, ce texte dessine plutôt les linéaments du "théâtre du quotidien " qui s'imposera dans les années 70 avec des dramaturges tels que Wenzel (Loin d'Hagondange, 1976), Lassalle (Un couple pour l'hiver, 1974) ou même Vinaver. Comme l'écrit le dramaturge Daniel Lemahieu, dans un tel théâtre « aucun sens, aucun destin ne préexistent à l'action même de parler. Ce théâtre se situe hors des valeurs constituées et ne délivre ni message, ni démonstration, ni engagement, ni modèle pour comprendre et transformer le monde. Les mots sont l'action. Ils ne sont pas le véhicule d'idées ou de sentiments clairement énoncés 5 ". Son dispositif scénique divisé invente de surcroît ce " théâtre de points de vue multiples » dont le théâtre du quotidien fera l'un de ses ressorts privilégiés.

La présentation que Wenzel donne de son projet semble ainsi dessiner $a$ posteriori la pièce de Claude Simon : "s'inscrire dans la banalité - enfouir les problèmes importants - loin dans la tête. Il ne s'agit pas d'une démonstration claire (les personnages ne se révoltent pas, ne dénoncent rien) ni de rendre compte du déroulement continu de la vie, mais d'un choix de moments où rien n'est dit explicitement et où surgissent le mieux l'aliénation et le refoulé de toute une $v^{6}{ }^{6} »$. Tout se joue bien dans les mots, dans leurs défaillances ou leur apparente banalité : "L'événement réside dans les creux, les anfrac-

4. Voir David Bradby, Le Théâtre français contemporain [Cambridge University Press, 1984], PU de Lille, 1990.

5. D. Lemahieu, article "Quotidien ", dans Michel Corvin (dir.), Dictionnaire encyclopédique du théâtre, Paris, L-Z, 1995, p. 742.

6. J.-P. Wenzel et C. Fiévet, «Pour un théâtre du quotidien ", dans Loin d'Hagondange. Marianne attend le mariage, Paris, Stock/Théâtre Ouvert, 1975, p. 9. 
tuosités de l'écriture laissant poindre des histoires multiples dont aucune ne prédomine. [...] Le personnage du théâtre quotidien est disloqué, en panne de mots, en manque de langue ou bien en excès de paroles fautives. Le personnage ne donne pas de leçons ", écrit encore Daniel Lemahieu. Telles sont bien, en effet, les figures élaborées par Claude Simon dans La Séparation.

Ce qui caractérise enfin La Séparation plus que tout sans doute et lui donne sa singularité en tant que pièce de théâtre, c'est son refus du spectacle et du spectaculaire. Texte et jeu restent au ras des choses, d'un quotidien certes dramatique (l'agonie d'une vieille femme qui a sacrifié sa vie; la mésentente des couples), mais banal au fond. Et aucun événement ne vient crever cette banalité. À rebours des effets et provocations de son temps, Simon ose un théâtre non théâtral, c'est-à-dire dépouillé aussi de ces " théâtralisations " comportementales qui fondent les métaphores et comparaisons critiques si nombreuses dans son œuvre que je rappelais au début de ce propos. Cette pièce interdit les effets spectaculaires, refuse tout recours à l'esthétisation, demeure au ras des choses et des individus, elle invite le spectateur à l'écoute des mots, des émotions, des intensités que ces mots refoulent et trahissent cependant.

Ce faisant, Simon anticipe sur les propos de Jacques Lassalle, qui postule " que l'exercice théâtral ne se situe pas sur le plan du spectaculaire, de l'apostrophe violente ". Le dramaturge explique "qu'il faut permettre à la représentation d'introduire très progressivement le spectateur dans une autre durée, un autre type d'écoute, une espèce d'état d'attention flottante, comme une invitation à entretenir avec son expérience du monde un autre rapport que celui proposé ici et là dans la représentation théâtrale ${ }^{8}$ ". N'est-ce pas de cela, justement, qu'il s'agit déjà dans La Séparation? Disposer le spectateur dans cette écoute particulière, où les réminiscences de Louise et de Georges, les évocations d'un rai de soleil qui se déplace lentement au fil du jour, le sentiment irrépressible du temps qui passe, de l'odeur des poires pourrissant et du regard devenu vide d'une femme à l'agonie, prennent tout à coup une singulière présence.

Ainsi, de même que l'on a pu récemment réfléchir à l'influence de Claude Simon romancier sur le travail des écrivains actuels ${ }^{9}$, il est sans doute néces-

7. D. Lemahieu, art. cit.

8. J. Lassalle, "À la renverse ", entretien avec Bernard Dort, Jacques Lassalle et Michel Vinaver, dans Théatrelpublic, no 32, mars 1980, p. 11.

9. Voir Maylis de Kerangal sur les grands chemins de Claude Simon, Carnets de Chaminadour, ${ }^{\circ}$ 10, 2017, notamment D. Viart, "Le legs de Claude Simon à la littérature contemporaine ", p. 167-185. 
saire de resituer La Séparation dans l'histoire du théâtre français. Certes, la pièce, isolée dans l'œuvre, et demeurée sans écho majeur, n'a pas véritablement "influencé » les dramaturges de la génération suivante. Même si certains de ces dramaturges connaissent l'œuvre de Simon, ce serait lui faire trop d'honneur que de le prétendre. Mais il est remarquable qu'elle anticipe des préoccupations et des choix dramaturgiques à venir. Elle manifeste ainsi la sensibilité toute particulière de Simon et, à certains égards, s'articule à la dimension humaniste de ses photographies, que l'on peut inscrire, elles, dans le droit fil des œuvres de Cartier-Bresson, de Boubat ou de Willy Ronis. Il est regrettable sans doute qu'une erreur de distribution et de jeu en ait très certainement faussé la réception lorsqu'elle fut présentée en 1963; mais il le serait encore plus que le contresens de la postface échoue à mettre en évidence le talent précurseur de ce beau texte. 